\title{
Second-order topological modes in two-dimensional continuous media
}

\author{
Jan Košata 1 and Oded Zilberberg \\ Institute for Theoretical Physics, ETH Zürich, 8093 Zürich, Switzerland
}

(Received 9 March 2021; accepted 28 June 2021; published 30 July 2021)

\begin{abstract}
We present a symmetry-based scheme to create zero-dimensional (0D) second-order topological modes in continuous two-dimensional (2D) systems. We show that a metamaterial with a $p 6 m$-symmetric pattern exhibits two Dirac cones, which can be gapped in two distinct ways by deforming the pattern. Combining the deformations in a single system then emulates the 2D Jackiw-Rossi model of a topological vortex, where 0D in-gap bound modes are guaranteed to exist. We exemplify our approach with the simple hexagonal, kagome, and honeycomb lattices. We furthermore formulate a quantitative method to extract the topological properties from finite-element simulations, which facilitates further optimization of the bound mode characteristics. Our scheme enables the realization of second-order topology in a wide range of experimental systems.
\end{abstract}

DOI: 10.1103/PhysRevResearch.3.L032029

The development of topological insulators (TIs), while originating in electronic systems, has made a profound impact in the field of classical metamaterials. Their signature topological boundary modes have been successfully demonstrated in photonic [1,2], electrical [3,4], phononic [5-7], acoustic [8,9], atomic [10], and polaritonic [11] systems. This remarkable universality stems from the origin of the boundary phenomena; these depend only on topological invariants derived from the bulk spectral bands, and not on the specific medium. Early realizations of topology in classical systems relied on engineering synthetic gauge fields in tight-binding (TB) models, obtained by coupling resonator modes in space [12,13] and/or time [14] to create topological boundary modes. Later it was discovered that in patterned continuous media, topologically distinct phases can be formed purely by breaking the spatial symmetries of the pattern [15]; this has opened the field of TIs to a wider range of platforms, and brought it closer to potential applications. Furthermore, the topological phases of matter paradigm has been recently extended to higher-order TIs (HOTIs) [16-24]. In a $d$-dimensional HOTI, topological modes of dimension $d-2$ and lower may appear. HOTIs have been experimentally demonstrated by emulating TB models in a variety of platforms [25-34].

The concepts of TIs and HOTIs are unified in the framework of ten topological classes, each characterized by time-reversal, chiral, and particle-hole symmetries [35]. For a $d$-dimensional system with a defect defined on a $\mathcal{D}$ dimensional surface, a topological invariant exists whose possible values depend only on the bulk symmetry class and $d-\mathcal{D}$ [36]. At such a defect, modes with dimension $d-1-\mathcal{D}$

Published by the American Physical Society under the terms of the Creative Commons Attribution 4.0 International license. Further distribution of this work must maintain attribution to the author(s) and the published article's title, journal citation, and DOI. (codimension $\mathcal{D}+1$ ) are formed. TIs, with their topology defined purely in their bulk, correspond to $\mathcal{D}=0$; for $\mathcal{D}>0$, the invariant generally includes integrals over curvatures combining real and momentum space, leading to the appearance of high-order topological modes [16-20,22-24,36] with topological boundary states of codimension $>1$. An instance of a HOTI is found in the $d=2$ Jackiw-Rossi Hamiltonian of a topological vortex, which can be implemented by deforming the TB model of graphene [37]. Emulating this model has successfully demonstrated the creation of zero-dimensional (0D) modes at topological vortices in photonic [38-40] and elastic [41] devices. Compared with standard OD modes formed at nontopological lattice defects, topological vortex modes offer several advantages [39]: (i) Their frequency is near midgap, resulting in better spatial confinement and quality factors. (ii) The number of modes formed is fixed by the topology. (iii) The modal area is scalable, as the modes form independently of the vortex size. These aspects make topological vortex modes promising candidates for the construction of singlemode semiconductor lasers.

In this Letter, we show how to obtain the Jackiw-Rossi Hamiltonian in continuous two-dimensional (2D) structures. Crucially, our approach does not require a recourse to a TB model or threading by magnetic fluxes. Instead, we utilize solely the breaking of spatial symmetries of a linear medium. We focus on the $p 6 m$ space group, picking three examples of metamaterial patterns: the simple hexagonal [Fig. 1(I)(a)], kagome [Fig. 1(II)(a)], and honeycomb [Fig. 1(III)(a)] lattices. Central to our work, the group $p 6 m$ has a 4D irreducible representation that manifests as two Dirac cones at the $K / K^{\prime}$ points in reciprocal space. We consider two distinct perturbations of the primitive cell, namely the breaking of inversion and translation symmetries, and find their matrix representations in the 4D eigenspace $[42,43]$. The perturbation matrices are shown to anticommute and thus correspond to different gap-opening terms; these constitute the Jackiw-Rossi model. By spatially varying the perturbations in a single system, a topological vortex defect is formed with in-gap OD modes 
(I)

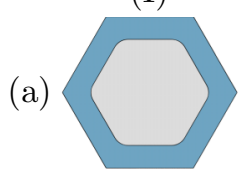

(b)

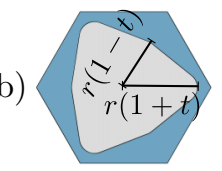

(c)

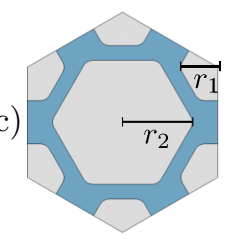

(II)
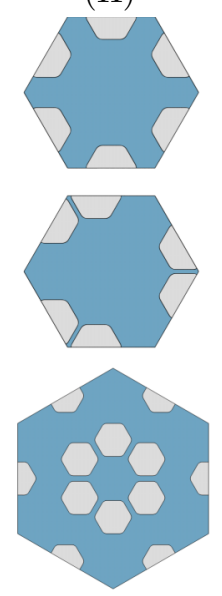

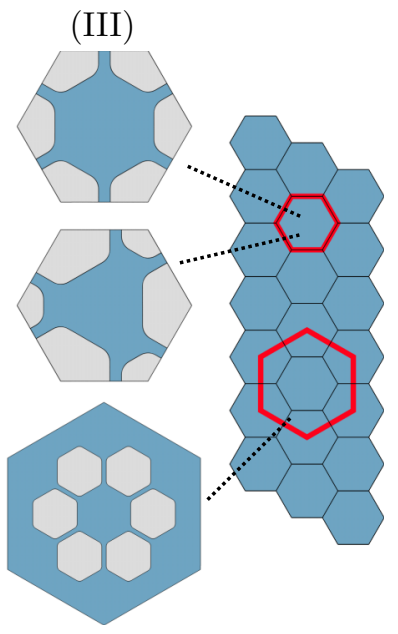

FIG. 1. Metamaterial unit cells for the continuum realization of the Jackiw-Rossi model [cf. Eq. (1)]. The cells are patterned by two different linear media, e.g., a dielectric (blue) with air incisions (gray). The three columns show (I) the simple hexagonal lattice, (II) the kagome lattice, and (III) the honeycomb lattice. The first row (a) corresponds to the starting unit cells, all of which form lattices with the $p 6 m$ space group. To obtain the anticommuting mass terms required in the Jackiw-Rossi model, we consider two symmetrybreaking perturbations; in row (b) breaking inversion symmetry [cf. Eq. (4)], and in row (c) breaking the translation symmetry of the primitive cell [cf. Eq. (5)]. This does not depend on the specific shapes of the incisions, as long as the respective symmetries are obeyed. The small and large red hexagons illustrate the unit cell before and after breaking translation symmetry. The labels $r, t, r_{1}, r_{2}$ in column (I) parametrize the incision shapes.

bound within. For concreteness, we assume a dielectric implementation of the scheme; we stress however that our analysis holds for any patterned 2D linear medium and is thus applicable to a wide range of systems.

The 2D Jackiw-Rossi Hamiltonian reads

$$
H=\boldsymbol{\gamma} \cdot \boldsymbol{k}+\boldsymbol{\Gamma} \cdot \boldsymbol{m}(\boldsymbol{r}),
$$

where $\boldsymbol{k}=\left(k_{x}, k_{y}\right)$ are quasimomenta, and $\boldsymbol{m}=\left(m_{1}, m_{2}\right)$ are mass terms that can change in space $\boldsymbol{r}=(x, y)$. The four $4 \times 4$ matrices, $\boldsymbol{\gamma}=\left(\gamma_{1}, \gamma_{2}\right)$ and $\boldsymbol{\Gamma}=\left(\Gamma_{1}, \Gamma_{2}\right)$, anticommute with one another. If either of the mass terms $m_{1}$ or $m_{2}$ is nonzero, the spectrum of $H$ is gapped around zero energy. We first consider the case where one mass term (e.g., $m_{2}$ ) vanishes and the other switches signs in space [e.g., $m_{1}=|m| \operatorname{sgn}(x)$ ], thus defining a domain wall at $x=0$. The distinct band topology of the two bulk phases then defines a topological defect $(\mathcal{D}=0)$ and a band inversion occurs across $x=0$. The domain wall can then be described by two copies of the Jackiw-Rebbi model $[15,44]$, and the system admits a $\mathbb{Z}_{2}$ invariant $[45,46]$. Correspondingly, two counterpropagating $1 \mathrm{D}$ edge states appear at the domain wall. Depending on the eigenbasis of the gapping term, we thus obtain the states associated with either the quantum spin Hall effect (QSHE) or the quantum valley Hall effect (QVHE).

Allowing instead both masses to vary over a spacedependent closed path defines a defect with $\mathcal{D}=1$ [36]. The Hamiltonian is then characterized by a $\mathbb{Z}$ topological invari- ant associated with the winding number $n$ of $\boldsymbol{m}(\boldsymbol{r})$. Taking $m_{1}+i m_{2}=|\boldsymbol{m}| e^{i \theta}$, the winding number is defined as

$$
n=\frac{1}{2 \pi} \oint_{S^{1}} d \theta,
$$

where $S^{1}$ denotes the closed path. At such a topological vortex, $n$ zero-dimensional bound modes are found. Systems with $|n|>0$ can be constructed within the TB model of graphene, where $m_{1}$ and $m_{2}$ are generated by spatial perturbations of the six-site unit cell [37]. We also emphasize that this model is topologically equivalent to other proposed TB models for HOTIs [23] and the invariant definition involves integrating over both real and momentum space, such that the winding encircles a quantized 4D Dirac cone [24].

Symmetry analysis. We focus on the $p 6 m$ space group, exemplified by the three unperturbed structures shown in Fig. 1(a). All three are symmetric under sixfold rotation $C_{6}$, reflection $\sigma_{x}$, and lattice translation $T$. The operations that leave the structures invariant form a group $\mathcal{G}$ that is isomorphic to $p 6 \mathrm{~m}$. The group $\mathcal{G}$ admits a $4 \mathrm{D}$ irreducible representation (irrep) derived from the little group of the $K / K^{\prime}$ points [47]. This irrep manifests itself as the familiar pair of Dirac cones, appearing at the valleys $K$ and $K^{\prime}$ in reciprocal space. Choosing a basis for this irrep defines a function $\rho(g)$, which maps each symmetry element $g \in \mathcal{G}$ to a $4 \times 4$ representation matrix $\rho(g)$. For the three group generators, we obtain [48]

$$
\begin{aligned}
& \rho\left(C_{6}\right)=\tau_{1} \otimes R_{6}, \quad \rho\left(\sigma_{x}\right)=\tau_{1} \otimes \tau_{3}, \\
& \rho(T)=\operatorname{diag}\left(w^{2}, w\right) \otimes \mathbb{1}_{2},
\end{aligned}
$$

where $\tau_{i}$ are Pauli matrices, $R_{6}$ denotes a $2 \times 2$ rotation matrix of angle $\pi / 3$, and $w=\exp \{2 \pi i / 3\}$. The basis is chosen such that each $\rho(g)$ is a direct product of a $2 \times 2$ matrix acting on the two valley degrees of freedom with another $2 \times 2$ matrix acting on the two degenerate bands within each valley.

Opening band gaps in the degenerate cones to create topologically distinct bulks can be achieved in two different ways: breaking inversion or translation symmetry, as shown in Figs. 1(b) and 1(c), respectively. Starting with the former, we break inversion symmetry while preserving $C_{3}$; this entails different deformations in each of the three example structures [see Fig. 1(b)]. These deformations leave the symmetry subgroup generated by $\left\{C_{3}, \sigma_{x}, T\right\}$ intact; the resulting space group is isomorphic to $p 3 m$, and the corresponding symmetrybreaking perturbation takes the form [48]

$$
M_{1}=c_{1} \mathbb{1}_{4}+m_{1} \Gamma_{1},
$$

where $\Gamma_{1}=\tau_{3} \otimes \tau_{2}$, and $c_{1}$ and $m_{1}$ are real coefficients that quantify the strength of the perturbation. Breaking translation symmetry, instead, we consider an enlarged realspace unit cell [Fig. 1(c)], which leads to band folding into a smaller Brillouin zone (BZ) in reciprocal space. The larger unit cell maps both valleys $K / K^{\prime}$ to the new $\Gamma$ point. Applying the appropriate deformation for each lattice then breaks the original translation invariance so that the system is no longer self-coincident under $T$. The remaining symmetry subgroup is generated by $\left\{C_{6}, \sigma_{x}, T C_{6} T\right\}$, where $T C_{6} T$ is the expanded lattice translation. The corresponding translation symmetry-breaking perturbation 
matrix is [48]

$$
M_{2}=c_{2} \mathbb{1}_{4}+m_{2} \Gamma_{2},
$$

with $\Gamma_{2}=\tau_{1} \otimes \mathbb{1}_{2}$, and real coefficients $c_{2}, m_{2}$.

The perturbation matrices $\Gamma_{1}$ and $\Gamma_{2}$ anticommute. Hence, when the band gaps of the two perturbed structures coincide in energy (i.e., when $c_{1}=c_{2}$ ), the identity terms in Eqs. (4) and (5) can be absorbed into the unperturbed Hamiltonian, leaving us with two anticommuting mass terms gapping the $K / K^{\prime}$ valleys. Note that while the enlarged unit cell is not primitive in the case of inversion-breaking $M_{1}$, choosing such a cell allows both perturbations to be combined in a single lattice. To complete the construction, we study the dispersion away from the two valleys; quasimomenta away from $K / K^{\prime}$ similarly result in perturbative terms, entering Eq. (1) as $[42,48,49]$

$$
\gamma_{1}=\tau_{3} \otimes \tau_{1}, \quad \gamma_{2}=\tau_{3} \otimes \tau_{3} .
$$

The two matrices $\gamma_{1}$ and $\gamma_{2}$ anticommute with each other and with both $\Gamma_{1}$ and $\Gamma_{2}$. As the symmetry-breaking perturbations may be position dependent, we have effectively arrived at the term $\boldsymbol{\Gamma} \cdot \boldsymbol{m}(\boldsymbol{r})$ and fully identified the ingredients for realizing the Jackiw-Rossi Hamiltonian in a continuous 2D medium.

We highlight that our analysis is applicable to a broad range of geometries and systems, as it relies solely on the symmetries of the perturbations and on time reversal. Moreover, our construction does not rely on introducing pseudospin or pseudo-time-reversal symmetries [15]. Instead, the initial fourfold degeneracy is directly obtained by starting with the original unit cell and its associated space group. We can thus also directly verify that our construction falls into the BDI class: In our chosen 4D basis, the time-reversal operator reads $\mathcal{T}=I \mathcal{K}$, where $I=\tau_{1} \otimes \mathbb{1}_{2}$ corresponds to the spatial inversion operator and $\mathcal{K}$ is the complex conjugation operator; chiral symmetry is given by $\mathcal{C}=\tau_{2} \otimes \mathbb{1}_{2}$. The Hamiltonian obeys these symmetries due to the spatial symmetries required by our construction. Since $\mathcal{C}^{2}=\mathcal{T}^{2}=1$, our system belongs to the BDI class $[35,36]$ and thus admits a topological winding number [cf. Eq. (2)].

The signs of $m_{1}$ and $m_{2}$ distinguish four topologically distinct phases [48]. We offer an intuitive view of this distinction by examining the induced band inversions in the expanded unit cell of the simple hexagonal lattice. We focus on the symmetries at the $\Gamma$ point and consider the point group $C_{3 v}$. We also choose bases for two instances of the $C_{3 v}$ twofold degenerate representation, e.g., the pairs of orbitals $\left\{p_{x}, p_{y}\right\}$ and $\left\{d_{x y}, d_{x^{2}-y^{2}}\right\}$. These can mix freely under $C_{3 v}$ symmetry, and are guaranteed to form a fourfold degeneracy when the original unit cell is intact. Under the perturbation $M_{1}$ (Fig. 2, upper panel), the two distinct orientations of the enlarged unit cell are mapped into each other by inversion, fixing the eigenspaces $\left\{p_{x}+d_{x y}, p_{y}+i d_{x^{2}-y^{2}}\right\}$ and $\left\{p_{x}-d_{x y}, p_{y}-i d_{x^{2}-y^{2}}\right\}$; these match realizations of the QVHE [50,51]. Under the perturbation $M_{2}$ (Fig. 2, lower panel), the enlarged unit cell remains inversion symmetric, so that its eigenspaces must consist of even and odd functions, here $\left\{p_{x}, p_{y}\right\}$ and $\left\{d_{x y}, d_{x^{2}-y^{2}}\right\}$, matching the crystalline realizations of the QSHE [15].

Bulk signatures. Our discussion has so far relied on general symmetry arguments. At the same time, we show in Fig. 1 examples of lattices and their deformations. In each structure,

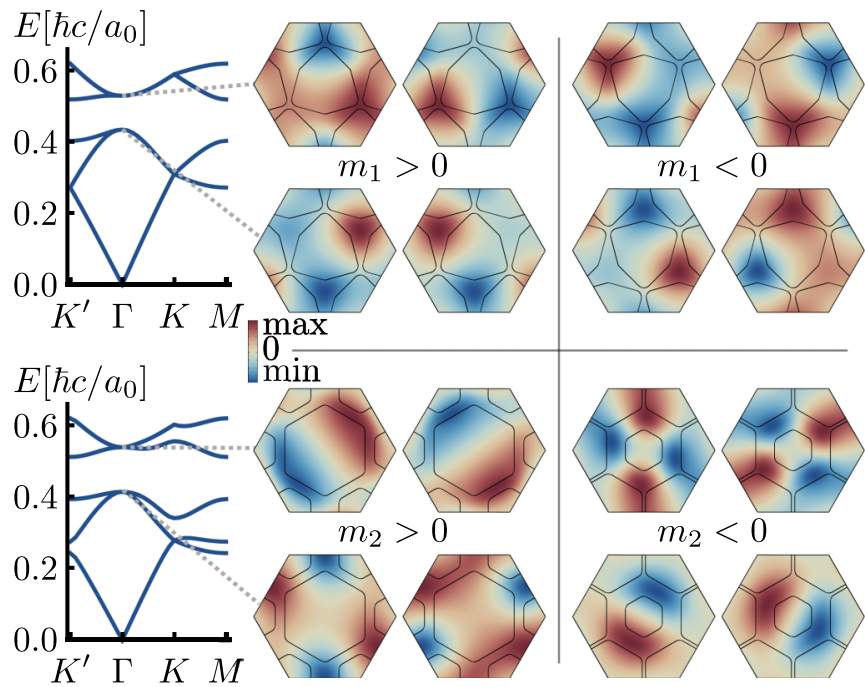

FIG. 2. The bulk solutions of the perturbed simple hexagonal lattice using the enlarged unit cell with periodic boundary conditions [cf. Fig. 1(I)]. The bulk spectrum and the four bulk eigenstates at the $\Gamma$ point below and above the gap for each perturbed unit cell structure, with positive/negative values for each of the symmetry-breaking mass terms. Top: Breaking inversion symmetry [cf. Fig. 1(b) and Eq. (4)]. Bottom: Breaking translation symmetry [cf. Fig. 1(c) and Eq. (5)].

the magnitudes of the mass terms $m_{1}$ and $m_{2}$ are evaluated numerically as they depend on the specifics of the symmetrybreaking deformations and material/platform realization. To extract the mass terms from the numerics, we focus on the $\Gamma$ point of the enlarged unit cell and obtain the four numerical solutions $H\left|\psi_{i}\right\rangle=E_{i}\left|\psi_{i}\right\rangle$, with energies $E_{i}$ and corresponding deviations from midgap $\Delta E_{i}=E_{i}-\bar{E}$ for $\bar{E}=\sum_{i} E_{i} / 4$. In the effective $4 \times 4$ model, the mass terms are easily evaluated, since $m_{i}=\left\langle\psi_{j}\left|\Gamma_{i}\right| \psi_{j}\right\rangle$. However, as seen in Fig. 2, the numerical solutions are obtained in a real-space basis. We therefore need to convert the matrices $\Gamma_{1}$ and $\Gamma_{2}$ to real-space operations, i.e., invert the function $\rho(g)$ in Eq. (3). Averaging over the four solutions then yields

$$
m_{i}=\sum_{j} \Delta E_{j}\left\langle\psi_{j}\left|\rho^{-1}\left(\Gamma_{i}\right)\right| \psi_{j}\right\rangle / 4,
$$

where we find using Eq. (3)

$$
\begin{aligned}
& \rho^{-1}\left(\Gamma_{1}\right)=-\left(4 C_{3} T+2 C_{3}+2 T+\mathbb{1}\right) / 3, \\
& \rho^{-1}\left(\Gamma_{2}\right)=-C_{6}^{3} .
\end{aligned}
$$

In Fig. 3, we plot the dependence of the effective masses $m_{i}$ on the structural features of the simple hexagonal lattice example [cf. Fig. 1(I)]. Here, inversion symmetry is broken by deforming the hexagonal incision into a triangle, parametrized by $t$. Translation symmetry is broken by introducing two different radii $r_{1}$ and $r_{2}$ for the central and outer hexagonal incisions, parametrized by $r_{1} / r_{2}$. Using such a construction, the deformations can be combined in a single unit cell [cf. Figs. 3(1)-3(4)]. The calculations are performed using COMSOL [52], and the mass terms are evaluated using Eq. (7). Crucially, the mass terms are varied 


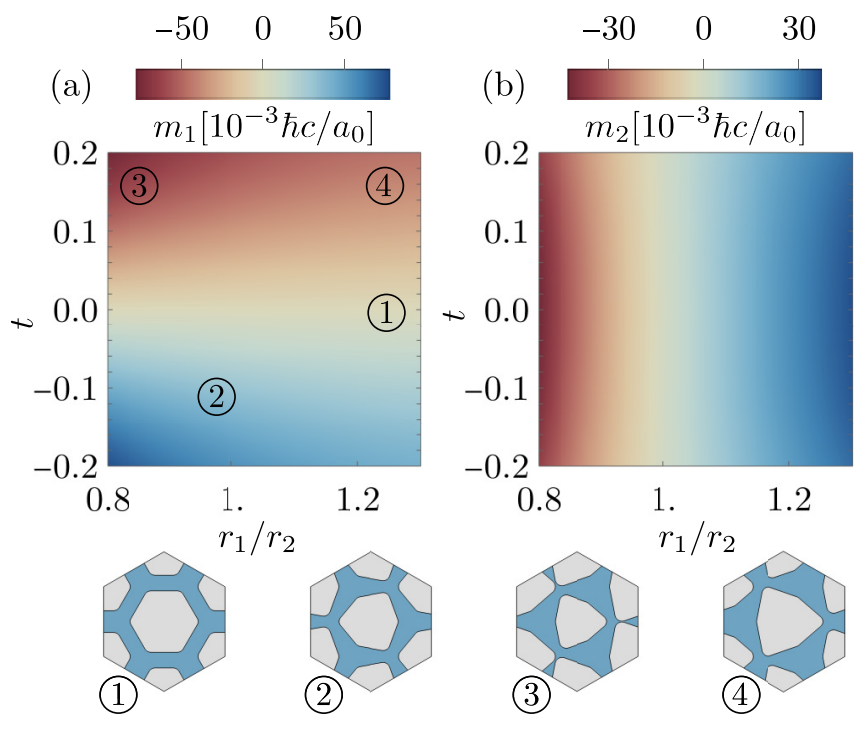

FIG. 3. The bulk solutions of the perturbed simple hexagonal lattice using the enlarged unit cell and combining the symmetrybreaking deformations [cf. Fig. 1(I)]. The (a) $m_{1}$ and (b) $m_{2}$ mass terms, extracted using Eq. (7). The bottom panel shows the unit cell at selected points in the configuration space.

independently by the different symmetry-breaking deformations [see Figs. 3(a) and 3(b)]. This confirms that we can readily realize a spatially dependent mass term $\boldsymbol{m}(\boldsymbol{r})$, as required by the Jackiw-Rossi model. We reiterate that to create a bound mode, the structures used for the winding must have coincident band gaps, analogously to $c_{1}=c_{2}$ in Eqs. (4) and (5).

We move now to construct a Jackiw-Rossi vortex in a large supercell composed of the different bulk phases. To obtain a topological bound mode, the mass vector must wind [cf. Eq. (2)], i.e., we need to spatially pass through all four "pure" perturbations shown in Fig. 2. Constructing a vortex of $n=1$ in a dielectric medium, we numerically confirm the existence of a bound mode in Fig. 4. To assure an overall band gap, we use only the four unit cells shown in Fig. 2. Since we use periodic boundary conditions, the supercell features four distinct vortex sites, all with the same $|n|$ but with different terminations. Correspondingly, we find four bound modes, each localized at one of the four vortices. By construction, the topological vortex modes should appear midgap. One of the vortex modes indeed lies at the bulk gap center, while the remaining three deviate slightly away [see Fig. 4(a)]. We attribute this discrepancy to the inequivalent vortex terminations, where the spatial symmetries are locally broken [39]. Note that gapped topological edge states exist at the domain walls between different mass regions, as expected in second-order topological insulators $[17,18,24]$ [see Figs. 4(a) and 4(c)].

Discussion. The presented symmetry principles and formulas for numerical calculations enable a systematic exploration of a plethora of structures, which feature high-order topological defects. The range of compatible structures is far broader than with the TB approach, e.g., the primitive cell of the

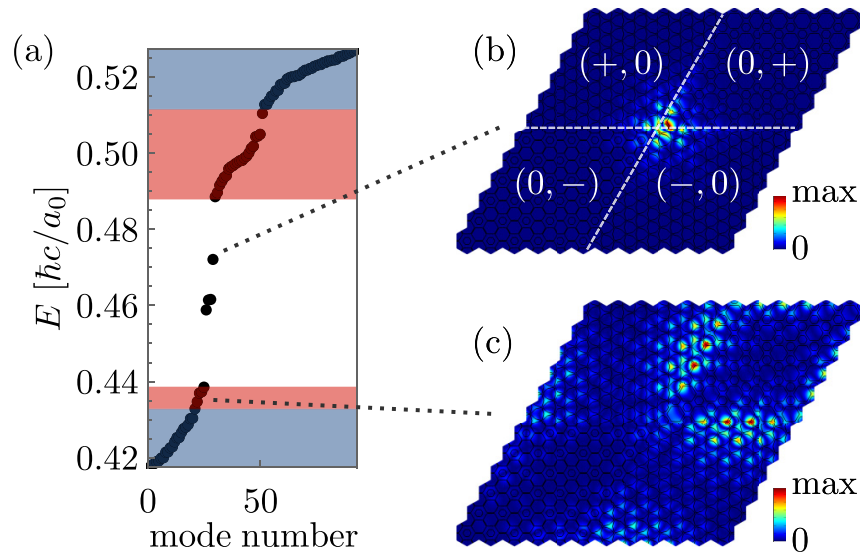

FIG. 4. Numerical simulation of a Jackiw-Rossi vortex with $n=$ 1 in a continuous dielectric $\left(\epsilon_{r}=11.7\right)$ patterned with air incisions. The simulated supercell combines four bulks corresponding to the four perturbed structures (1)-(4) in Fig. 3. This gives rise to a JackiwRossi vortex (1) at the cell's center. Periodic boundary conditions are used at all four edges of the cell, giving a total of four vortices. (a) The energy spectrum of the structure showing bulk modes (blue fill), edge modes (red fill), and OD bound modes (white fill). (b) The supercell, showing the signs of the mass terms $\left(m_{1}, m_{2}\right)$ and boundaries between the phases. The amplitude of a midgap solution is overlayed onto the structure, exhibiting a mode bound at the center. (c) A similar plot for one of the gapped edge mode solutions.

simple hexagonal (kagome) lattice has one (three) incisions, which precludes the formulation of a four-band TB model required by a $2 \mathrm{D}$ HOTI. At the same time, we emphasize that the symmetry arguments describe the behavior of the bands near the high-symmetry points of the BZ, but do not guarantee a fully gapped spectrum. In the kagome lattice, for example, a dielectric patterned with air incisions shows the correct symmetry breaking at the $\Gamma$ point, but the spectrum is gapless due to the band dispersion at other regions of the BZ, yielding only an indirect gap at the $\Gamma$ point. Such limitations are peculiar to specific experimental platforms. From a technological standpoint, the bulk band gap size is the key characteristic to keeping the vortex modes spectrally isolated and spatially confined. In Fig. 4(a), its size relative to the gap center is $16.6 \%$, which is a factor of 3 higher compared to existing works based on the graphene-inspired TB model [39]. Further improvement is expected with the use of numerical optimization enabled by our work. We also note that the spatial confinement may be strengthened by nonlinear interactions [53,54]. Finally, we highlight that our scheme can be extended to three dimensions by appropriately extruding the geometry in the out-of-plane direction. This yields one-dimensional, counterpropagating topological modes that can be used to construct optical fibers [55,56].

Acknowledgments. This work was supported by the Swiss National Science Foundation through Grant CRSII5 177198/1, PP00P2_163818. We also thank I. Petrides, M. Rechtsman, T. Wolf, A. Eichler, and R. Chitra for helpful discussions. 
[1] F.-F. Li, H.-X. Wang, Z. Xiong, Q. Lou, P. Chen, R.-X. Wu, Y. Poo, J.-H. Jiang, and S. John, Topological light-trapping on a dislocation, Nat. Commun. 9, 2462 (2018).

[2] T. Ozawa, H. M. Price, A. Amo, N. Goldman, M. Hafezi, L. Lu, M. C. Rechtsman, D. Schuster, J. Simon, O. Zilberberg, and I. Carusotto, Topological photonics, Rev. Mod. Phys. 91, 015006 (2019).

[3] J. Ningyuan, C. Owens, A. Sommer, D. Schuster, and J. Simon, Time- and Site-Resolved Dynamics in a Topological Circuit, Phys. Rev. X 5, 021031 (2015).

[4] T. Hofmann, T. Helbig, C. H. Lee, M. Greiter, and R. Thomale, Chiral Voltage Propagation and Calibration in a Topolectrical Chern Circuit, Phys. Rev. Lett. 122, 247702 (2019).

[5] L. M. Nash, D. Kleckner, A. Read, V. Vitelli, A. M. Turner, and W. T. M. Irvine, Topological mechanics of gyroscopic metamaterials, Proc. Natl. Acad. Sci. USA 112, 14495 (2015).

[6] S. H. Mousavi, A. B. Khanikaev, and Z. Wang, Topologically protected elastic waves in phononic metamaterials, Nat. Commun. 6, 8682 (2015).

[7] S. D. Huber, Topological mechanics, Nat. Phys. 12, 621 (2016).

[8] C. He, X. Ni, H. Ge, X.-C. Sun, Y.-B. Chen, M.-H. Lu, X.-P. Liu, and Y.-F. Chen, Acoustic topological insulator and robust one-way sound transport, Nat. Phys. 12, 1124 (2016).

[9] G. Ma, M. Xiao, and C. T. Chan, Topological phases in acoustic and mechanical systems, Nat. Rev. Phys. 1, 281 (2019).

[10] N. R. Cooper, J. Dalibard, and I. B. Spielman, Topological bands for ultracold atoms, Rev. Mod. Phys. 91, 015005 (2019).

[11] M. Milićević, T. Ozawa, P. Andreakou, I. Carusotto, T. Jacqmin, E. Galopin, A. Lemaitre, L. Le Gratiet, I. Sagnes, and J. Bloch, Edge states in polariton honeycomb lattices, 2D Mater. 2, 034012 (2015).

[12] Y. E. Kraus, Y. Lahini, Z. Ringel, M. Verbin, and O. Zilberberg, Topological States and Adiabatic Pumping in Quasicrystals, Phys. Rev. Lett. 109, 106402 (2012).

[13] M. Hafezi, S. Mittal, J. Fan, A. Migdall, and J. Taylor, Imaging topological edge states in silicon photonics, Nat. Photonics 7, 1001 (2013).

[14] M. C. Rechtsman, J. M. Zeuner, Y. Plotnik, Y. Lumer, D. Podolsky, F. Dreisow, S. Nolte, M. Segev, and A. Szameit, Photonic Floquet topological insulators, Nature (London) 496, 196 (2013).

[15] L.-H. Wu and X. Hu, Scheme for Achieving a Topological Photonic Crystal by using Dielectric Material, Phys. Rev. Lett. 114, 223901 (2015).

[16] Y. E. Kraus, Z. Ringel, and O. Zilberberg, Four-Dimensional Quantum Hall Effect in a Two-Dimensional Quasicrystal, Phys. Rev. Lett. 111, 226401 (2013).

[17] W. A. Benalcazar, B. A. Bernevig, and T. L. Hughes, Electric multipole moments, topological multipole moment pumping, and chiral hinge states in crystalline insulators, Phys. Rev. B 96, 245115 (2017).

[18] W. A. Benalcazar, B. A. Bernevig, and T. L. Hughes, Quantized electric multipole insulators, Science 357, 61 (2017).

[19] I. Petrides, H. M. Price, and O. Zilberberg, Six-dimensional quantum Hall effect and three-dimensional topological pumps, Phys. Rev. B 98, 125431 (2018).

[20] O. Zilberberg, S. Huang, J. Guglielmon, M. Wang, K. P. Chen, Y. E. Kraus, and M. C. Rechtsman, Photonic topological boundary pumping as a probe of 4D quantum Hall physics, Nature (London) 553, 59 (2018).
[21] W. A. Benalcazar, T. Li, and T. L. Hughes, Quantization of fractional corner charge in $C_{n}$-symmetric higher-order topological crystalline insulators, Phys. Rev. B 99, 245151 (2019).

[22] D. Călugăru, V. Juričić, and B. Roy, Higher-order topological phases: A general principle of construction, Phys. Rev. B 99, 041301(R) (2019).

[23] T. Fukui, Dirac fermion model associated with a second-order topological insulator, Phys. Rev. B 99, 165129 (2019).

[24] I. Petrides and O. Zilberberg, Higher-order topological insulators, topological pumps and the quantum Hall effect in high dimensions, Phys. Rev. Research 2, 022049(R) (2020).

[25] S. Imhof, C. Berger, F. Bayer, J. Brehm, L. W. Molenkamp, T. Kiessling, F. Schindler, C. H. Lee, M. Greiter, T. Neupert et al., Topolectrical-circuit realization of topological corner modes, Nat. Phys. 14, 925 (2018).

[26] J. Noh, W. A. Benalcazar, S. Huang, M. J. Collins, K. P. Chen, T. L. Hughes, and M. C. Rechtsman, Topological protection of photonic mid-gap defect modes, Nat. Photonics 12, 408 (2018).

[27] F. Schindler, A. M. Cook, M. G. Vergniory, Z. Wang, S. S. Parkin, B. A. Bernevig, and T. Neupert, Higher-order topological insulators, Sci. Adv. 4, eaat0346 (2018).

[28] M. Serra-Garcia, V. Peri, R. Süsstrunk, O. R. Bilal, T. Larsen, L. G. Villanueva, and S. D. Huber, Observation of a phononic quadrupole topological insulator, Nature (London) 555, 342 (2018).

[29] H. Fan, B. Xia, L. Tong, S. Zheng, and D. Yu, Elastic Higher-Order Topological Insulator with Topologically Protected Corner States, Phys. Rev. Lett. 122, 204301 (2019).

[30] H. Xue, Y. Yang, F. Gao, Y. Chong, and B. Zhang, Acoustic higher-order topological insulator on a kagome lattice, Nat. Mater. 18, 108 (2019).

[31] B.-Y. Xie, H.-F. Wang, H.-X. Wang, X.-Y. Zhu, J.-H. Jiang, M.-H. Lu, and Y.-F. Chen, Second-order photonic topological insulator with corner states, Phys. Rev. B 98, 205147 (2018).

[32] X. Zhang, H.-X. Wang, Z.-K. Lin, Y. Tian, B. Xie, M.-H. Lu, Y.-F. Chen, and J.-H. Jiang, Second-order topology and multidimensional topological transitions in sonic crystals, Nat. Phys. 15, 582 (2019)

[33] X. Zhang, Z.-K. Lin, H.-X. Wang, Z. Xiong, Y. Tian, M.-H. Lu, Y.-F. Chen, and J.-H. Jiang, Symmetry-protected hierarchy of anomalous multipole topological band gaps in nonsymmorphic metacrystals, Nat. Commun. 11, 65 (2020).

[34] X. Zhou, Z.-K. Lin, W. Lu, Y. Lai, B. Hou, and J.-H Jiang, Twisted quadrupole topological photonic crystals, Laser Photonics Rev. 14, 2070046 (2020).

[35] C.-K. Chiu, J. C. Y. Teo, A. P. Schnyder, and S. Ryu, Classification of topological quantum matter with symmetries, Rev. Mod. Phys. 88, 035005 (2016).

[36] J. C. Y. Teo and C. L. Kane, Topological defects and gapless modes in insulators and superconductors, Phys. Rev. B 82, 115120 (2010).

[37] C.-Y. Hou, C. Chamon, and C. Mudry, Electron Fractionalization in Two-Dimensional Graphenelike Structures, Phys. Rev. Lett. 98, 186809 (2007).

[38] P. Gao, D. Torrent, F. Cervera, P. San-Jose, J. Sánchez-Dehesa, and J. Christensen, Majorana-Like Zero Modes in Kekulé Distorted Sonic Lattices, Phys. Rev. Lett. 123, 196601 (2019).

[39] X. Gao, L. Yang, H. Lin, L. Zhang, J. Li, F. Bo, Z. Wang, and L. Lu, Dirac-vortex topological cavities, Nat. Nanotechnol. 15, 1012 (2020). 
[40] Y. Yang, Z. Jia, Y. Wu, R.-C. Xiao, Z. H. Hang, H. Jiang, and $\mathrm{X}$. Xie, Gapped topological kink states and topological corner states in honeycomb lattice, Sci. Bull. 65, 531 (2020).

[41] X. Wu, Y. Meng, Y. Hao, R.-Y. Zhang, J. Li, and X. Zhang, Topological Corner Modes Induced by Dirac Vortices in Arbitrary Geometry, Phys. Rev. Lett. 126, 226802 (2021).

[42] M. Saba, S. Wong, M. Elman, S. S. Oh, and O. Hess, Nature of topological protection in photonic spin and valley Hall insulators, Phys. Rev. B 101, 054307 (2020).

[43] J. Cano, B. Bradlyn, Z. Wang, L. Elcoro, M. G. Vergniory, C. Felser, M. I. Aroyo, and B. A. Bernevig, Building blocks of topological quantum chemistry: Elementary band representations, Phys. Rev. B 97, 035139 (2018).

[44] R. Jackiw and C. Rebbi, Solitons with fermion number $1 / 2$, Phys. Rev. D 13, 3398 (1976).

[45] C. L. Kane and E. J. Mele, $Z_{2}$ Topological Order and the Quantum Spin Hall Effect, Phys. Rev. Lett. 95, 146802 (2005).

[46] X.-L. Qi, T. L. Hughes, and S.-C. Zhang, Topological field theory of time-reversal invariant insulators, Phys. Rev. B 78, 195424 (2008).

[47] C. Bradley and A. Cracknell, The Mathematical Theory of Symmetry in Solids: Representation Theory for Point Groups and Space Groups (Oxford University Press, Oxford, U.K., 2009).
[48] See Supplemental Material at http://link.aps.org/supplemental/ 10.1103/PhysRevResearch.3.L032029 for the derivations of the representation matrices and symmetry-allowed perturbative terms.

[49] M. Saba, J. M. Hamm, J. J. Baumberg, and O. Hess, Group Theoretical Route to Deterministic Weyl Points in Chiral Photonic Lattices, Phys. Rev. Lett. 119, 227401 (2017).

[50] T. Ma and G. Shvets, All-Si valley-Hall photonic topological insulator, New J. Phys. 18, 025012 (2016).

[51] J. Lu, C. Qiu, L. Ye, X. Fan, M. Ke, F. Zhang, and Z. Liu, Observation of topological valley transport of sound in sonic crystals, Nat. Phys. 13, 369 (2017).

[52] COMSOL MULTIPHYSICS v. 5.5, https://www.comsol.com/ (2019).

[53] D. Smirnova, D. Leykam, Y. Chong, and Y. Kivshar, Nonlinear topological photonics, Appl. Phys. Rev. 7, 021306 (2020).

[54] Y. E. Kraus, O. Zilberberg, and R. Berkovits, Enhanced compressibility due to repulsive interaction in the Harper model, Phys. Rev. B 89, 161106(R) (2014).

[55] H. Lin and L. Lu, Dirac-vortex topological photonic crystal fibre, Light Sci. Appl. 9, 202 (2020).

[56] L. Lu, H. Gao, and Z. Wang, Topological one-way fiber of second Chern number, Nat. Commun. 9, 5384 (2018). 